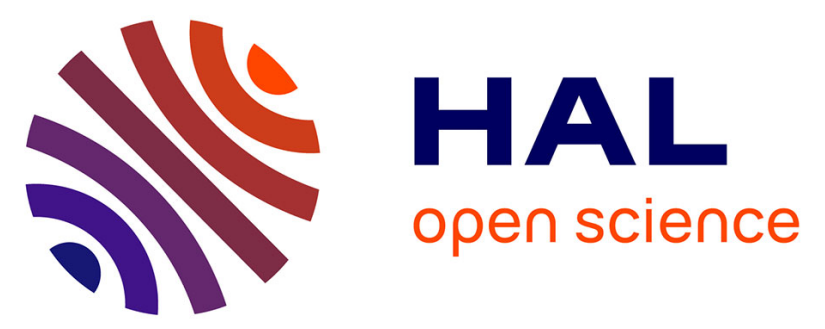

\title{
Optimal Control for Whole-Body Motion Generation using Center-of-Mass Dynamics for Predefined Multi-Contact Configurations
}

M Kudruss, Maximilien Naveau, Olivier Stasse, Nicolas Mansard, C Kirches, Philippe Souères, K Mombaur

\section{To cite this version:}

M Kudruss, Maximilien Naveau, Olivier Stasse, Nicolas Mansard, C Kirches, et al.. Optimal Control for Whole-Body Motion Generation using Center-of-Mass Dynamics for Predefined Multi-Contact Configurations. IEEE-RAS International Conference on Humanoid Robots (HUMANOIDS 2015), Nov 2015, Séoul, South Korea. 7p. hal-01179667v2

\section{HAL Id: hal-01179667 \\ https://hal.science/hal-01179667v2}

Submitted on 28 Jun 2016

HAL is a multi-disciplinary open access archive for the deposit and dissemination of scientific research documents, whether they are published or not. The documents may come from teaching and research institutions in France or abroad, or from public or private research centers.
L'archive ouverte pluridisciplinaire HAL, est destinée au dépôt et à la diffusion de documents scientifiques de niveau recherche, publiés ou non, émanant des établissements d'enseignement et de recherche français ou étrangers, des laboratoires publics ou privés. 


\title{
Optimal Control for Whole-Body Motion Generation using Center-of-Mass Dynamics for Predefined Multi-Contact Configurations
}

\author{
M. Kudruss ${ }^{1 \dagger}$, M. Naveau ${ }^{2 \dagger}$, O. Stasse ${ }^{2}$, N. Mansard ${ }^{2}$, C. Kirches ${ }^{1}$, P. Souères ${ }^{2}$, K. Mombaur ${ }^{1}$
}

\begin{abstract}
Multi-contact motion generation is an important problem in humanoid robotics because it generalizes bipedal locomotion and thus expands the functional range of humanoid robots. In this paper, we propose a complete solution to compute a fully-dynamic multi-contact motion of a humanoid robot. We decompose the motion generation by computing first a dynamically-consistent trajectory of the center of mass of the robot and finding then the whole-body movement following this trajectory. A simplified dynamic model of the humanoid is used to find optimal contact forces as well as a kinematic feasible center-of-mass trajectory from a predefined series of contacts. We demonstrate the capabilities of the approach by making the real humanoid robot platform HRP-2 climb stairs with the use of a handrail. The experimental study also shows that utilization of the handrail lowers the power consumption of the robot by $\mathbf{2 5} \%$ compared to a motion, where only the feet are used.
\end{abstract}

\section{INTRODUCTION}

The generation of whole-body motions utilizing multiple contacts between the robot and its environment extend the form of bipedal locomotion with a potential high impact on humanoid robot functionalities. It enables a robot to climb ladders, perform crawling, evolve in cluttered environment and less impressively, but yet very useful, to climb stairs [1], [2]. Staircase climbing is an important basic behavior for humanoid robots aiming at evolving in an industrial environment (see Fig. 1). The DARPA Robotics challenge have illustrated the difficulty of its realization. Two main classes of approaches can be distinguished in the resolution of the multi-contact locomotion problem. On the one hand, the problem is approached all at once, by trying to find a complete trajectory of the system typically using a numerical resolution scheme. On the other hand, the problem is decomposed into several sub problems, typically by following the seminal approach used in ground-level biped locomotion.

Ideally multi-contact motion generation includes a dynamic model of the humanoid, a model of its actuators and takes into account all its constraints over a finite previewwindow. The number of degrees of freedom (DoFs) and the size of the needed preview-window makes this approach useful for motions [3] exploiting the whole robot, but their computational complexity prevent an execution in real-time.

This work was supported by the European project KOROIBOT FP7-ICT$2013-10 / 611909$. $\dagger$ The first two authors contributed equally to this work.

${ }^{1}$ Interdisciplinary Center for Scientific Computing (IWR), Heidelberg University, Im Neuenheimer Feld 368, 69120 Heidelberg, Germany. \{manuel.kudruss, christian.kirches, katja.mombaur\} @iwr. uni-heidelberg.de

${ }^{2}$ LAAS-CNRS, 7 av. du Colonel Roche, F-31400, Toulouse, France, Univ de Toulouse, LAAS, F-31400, Toulouse, France \{mnaveau, ostasse, nmansard, soueres\}elaas.fr

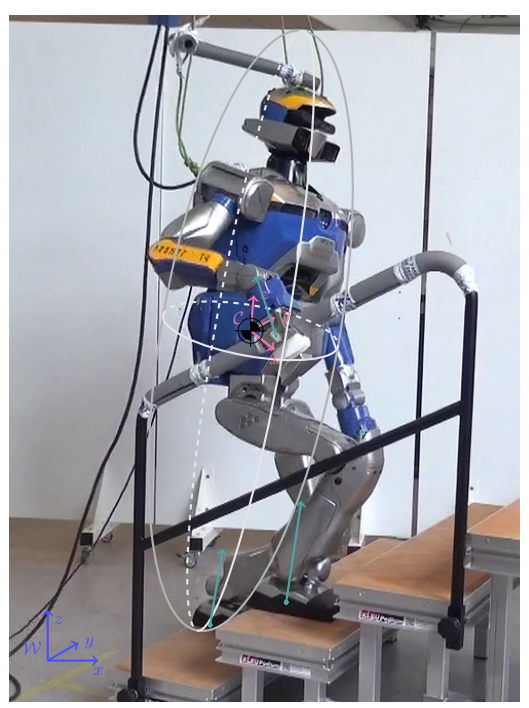

Fig. 1. HRP-2 climbing stairs with the support of the handrail. The overlay shows the reduced model (center of mass (CoM, black) at waist, CoM frame (magenta), gray inertia ellipsoid, contacts (teal dots) and contact contact forces (teal arrows) as well as world coordinate system $\mathscr{W}$ (blue).

The first class of approaches then mostly tries to make the problem tractable by proposing various ways of approximating the complete problem or improving the mathematical properties arising from a specific formulation. [1] proposed to work on the under-actuated dynamics and consider only the constraints related to inverse kinematics. [4] proposed to reformulate the contact model, and used differential dynamic programming to solve the related optimization problem efficiently. The same approach was implemented in [5] on the real HRP-2 robot for real-time control; however, no walking has been demonstrated yet on the real robot with this method.

Another approach is presented in [6], where they consider a set of contact points for which a dynamically consistent CoM trajectory is found. The forces are subject to the linearized friction cone constraints. This iterative algorithm assumes a predefined partitioning of the external forces applied to the system. In [7] a stabilization process based upon the work of Cheng [8] is proposed, which assumes a quasistatic motion, i.e. accelerations are set to zero. This condition imposes strong restrictions on the possible motions.

For ground-level walking the state of the art is the application of so called walking pattern generators that plan CoM trajectories for either predefined or additionally online computed foot contacts. Extensive simplifications of the dynamic model of the humanoid result in a linear inverted pendulum 
model, which allows the fast generation of walking motions. The extensions proposed in [9] can handle steep slopes of $10^{\circ}$ and demonstrate the humanoid robot platform HRP-2 [10] to even hit obstacles and adapt its feet trajectory appropriately. While these simplified models have a small computational footprint and show consistency with the elementary parts of the human gait, they all assume zero variations of the angular momentum about the CoM, which is once more a strong limitation for achieving complex dynamic movements.

These angular effects can be integrated based on the centroidal momentum described in [11]. Thereby, the robot is not only modeled as a point mass but by a spheroid with 3D inertia. A coordinate frame is attached to the CoM where the inertia is expressed. It is therefore possible to compute the variation of angular momentum created by contacts between the robot and its environment on the CoM.

\section{Contribution of the article}

In this work, we present a complete solution to generate whole-body motions for multi-contact supports considering a template model of the humanoid. We present a mathematical formulation of the reduced multi-contact CoM dynamics of an humanoid as an optimal control problem (OCP). The OCP is able to find feasible CoM trajectories and contact forces for predefined contact sets subject to constraints of respecting the contact model and constraints encompassing the kinematic limits of the whole-body system. The template model includes the major effects on the under-actuated part and is applicable for any combination of contact (ground level, biped walk on non-flat floor, multi-contact like using the handrail during stair climbing, etc). This approach is then used to generate a whole-body motion of climbing stairs with support of a handrail for the humanoid robot platform HRP-2. The experimental study on the robot show that handrail support reduces the motor power consumption by $25 \%$.

\section{Organization of the article}

We first describe the formulation of the OCP used to compute the CoM trajectory, and briefly discuss the particular numerical scheme used to solve this problem in Section II. In Section III, we explain how to process the CoM trajectory and contact forces to create a feasible motion for the humanoid. Finally, we will show the results we obtained from experiments on the real robot HRP-2 performing a stair climbing motion with hand rail support in section IV.

\section{GENERATION OF THE CENTER-OF-MASS TRAJECTORY}

\section{A. Dynamic model and constraints}

Inertia ellipsoid model: The derivation of the template model generalizes with the number of considered contact points $M \in \mathbb{N}$ located at positions $\mathbf{p}_{i} \in \mathbb{R}^{3}$, for $i=1 \ldots M$. The force applied at $\mathbf{p}_{i}$ is denoted by $\mathbf{f}_{i}=\left[f_{i, x} f_{i, y} f_{i, z}\right]^{\top}$, and is given in a local coordinate system, with the $z$-axis normal to the contact surface at $\mathbf{p}_{i}$. The component $f_{i, z}$ is the normal force applied at contact point $\mathbf{p}_{i}$ and $\left(f_{i, x}, f_{i, y}\right)$ is the tangential force applied at $\mathbf{p}_{i}$. However, we only use the end of the $M=3$ involved robot limbs for the representation of the stair climbing motion, i.e., the right hand $p_{r h}$ as well as the left foot $p_{l f}$ and right foot $p_{r f}$.

Following the ideas from [11], [9], we only consider the effect of the whole-body dynamics of a humanoid robot on the CoM. The centroidal dynamics is described as

$$
m\left[\begin{array}{c}
\ddot{\mathbf{c}}-\mathbf{g} \\
\mathbf{c} \times(\ddot{\mathbf{c}}-\mathbf{g})
\end{array}\right]+\left[\begin{array}{c}
\mathbf{0} \\
\dot{\mathscr{L}}
\end{array}\right]=\left[\begin{array}{c}
\sum_{i=1}^{M} \mathbf{Q}_{i} \mathbf{f}_{i} \\
\sum_{i=1}^{M} \mathbf{p}_{i} \times \mathbf{Q}_{i} \mathbf{f}_{i}
\end{array}\right]
$$

where $\mathbf{c}=\left[\begin{array}{lll}c_{x} & c_{y} & c_{z}\end{array}\right]^{\top} \in \mathbb{R}^{3}$ is the CoM and $m \in \mathbb{R}$ is the mass of the humanoid multi-body system, $\ddot{\mathbf{c}}=\left[\ddot{c}_{x} \ddot{c}_{y} \ddot{c}_{z}\right]^{\top} \in \mathbb{R}^{3}$ is the CoM acceleration, $\dot{\mathscr{L}}$ is the derivative of the robot angular momentum due to the angular speed of the robot body parts, and $\mathbf{Q}_{i} \in S O(3)$ is the $3 \times 3$ rotation matrix that transforms forces $\mathbf{f}_{i}$ in the local contact coordinate system at $\mathbf{p}_{i}$ into the global world coordinate system.

In the following the contribution to the variation of the angular momentum $\mathscr{L}$ is separated between the part caused by the angular acceleration of the whole robot body and the internal movements of the robot bodies $\dot{\mathscr{L}}=\mathbf{I}_{c} \dot{\omega}-\sigma$, where $\mathbf{I}_{c}$ is the inertia matrix of the whole body considered as a single rigid body computed for the configuration at time $t_{0}$, $\dot{\omega}$ is the angular acceleration (3D) of the root frame attached to the waist body of the robot, and $\sigma$ is a function of the whole-body configuration, velocity and acceleration [11] that does not depend on $\omega$. Eq. 1 is then rewritten in form of

$$
\mathbf{M}(\mathbf{c})\left[\begin{array}{c}
\ddot{\mathbf{c}} \\
\dot{\omega}
\end{array}\right]=\sum_{i} \mathbf{J}_{i}^{T} \mathbf{f}_{i}+\mathbf{S}^{T} \sigma+\mathbf{b}(\mathbf{c}),
$$

with

$$
\begin{array}{rlrl}
\mathbf{M}(\mathbf{c}) & =\left[\begin{array}{cc}
m \mathbf{1}_{3} & \mathbf{0}_{3 \times 3} \\
m \mathbf{c} \times & \mathbf{I}_{c}
\end{array}\right], & \mathbf{J}_{i}^{T} & =\left[\begin{array}{c}
\mathbf{Q}_{i} \\
\mathbf{p}_{i} \times \mathbf{Q}_{i}
\end{array}\right], \\
\mathbf{S}^{T} & =\left[\begin{array}{l}
\mathbf{0}_{3} \\
\mathbf{1}_{3}
\end{array}\right], & \mathbf{b}(\mathbf{c})=\left[\begin{array}{c}
m \mathbf{g} \\
m \mathbf{c} \times \mathbf{g}
\end{array}\right],
\end{array}
$$

where $\mathbf{c} \times \in \mathbb{R}^{3 \times 3}$ (resp. $\mathbf{p}_{i} \times$ ) is the skew matrix associated with vector $\mathbf{c}$ (resp. $\mathbf{p}_{i}$ ).

The equilibrium constraints (2) are rewritten according to [12] as non-linear first-order differential equation in form of

$$
\frac{d}{d t}\left[\begin{array}{c}
\mathbf{c} \\
\theta \\
\dot{\mathbf{c}} \\
\omega
\end{array}\right]=\left[\begin{array}{c}
\dot{\mathbf{c}} \\
\omega \\
(\mathbf{M}(\mathbf{c}))^{-1}\left(\sum_{i} \mathbf{J}_{i}^{T} \mathbf{f}_{i}+\mathbf{S}^{T} \sigma+\mathbf{b}(\mathbf{c})\right)
\end{array}\right] .
$$

Linear constraints: The applied contact model treats the feet contacts as unilateral and the hand contact on the rail as bilateral. This is achieved by defining proper bounds onto the applied contact forces given by

$\left[\begin{array}{c}-600 \\ -600 \\ 0\end{array}\right] \leq \mathbf{f}_{\mathbf{l f} / \mathbf{r f}} \leq\left[\begin{array}{c}600 \\ 600 \\ 600\end{array}\right],\left[\begin{array}{l}-150 \\ -150 \\ -150\end{array}\right] \leq \mathbf{f}_{\mathbf{r f}} \leq\left[\begin{array}{c}150 \\ 150 \\ 150\end{array}\right],\left[\begin{array}{l}-600 \\ -600 \\ -600\end{array}\right] \leq \sigma \leq\left[\begin{array}{c}600 \\ 600 \\ 600\end{array}\right]$

The bounds are manually chosen according to the experience of the experimenters. Contact forces can only be applied when a contact is established. When the contact is released 
TABLE I

OBJECTIVE WEIGHTS

\begin{tabular}{c|ccccccc}
\hline$i$ & 0 & 1 & 2 & 3 & 4 & 5 & 6 \\
\hline$w_{i}$ & 0.05 & 0.0005 & 1.0 & 1.0 & 1.00 & 0.1 & 1.00 \\
\hline
\end{tabular}

(or moving), we define the lower and upper bounds for the contact force to be zero. The description of a moving contact is denoted by $\left\|\dot{\mathbf{p}}_{i}\right\|_{2}>0$. We refer to the contact complementarity to indicate if the end effector is in contact or not given by

$$
\left\|\dot{\mathbf{p}}_{i}\right\|_{2} \cdot\left\|\mathbf{f}_{i}\right\|_{2}=0
$$

However, note that the complementarity is not explicitly treated yet, but is predefined in the contact configuration.

Friction cone constraints: The applied friction model requires the contact forces to satisfy the so called friction cone constraints, which are given for $M$ contact points by

$$
\left\|\left(f_{i, x}, f_{i, y}\right)\right\|_{2}=\sqrt{\left(f_{i, x}\right)^{2}+\left(f_{i, y}\right)^{2}} \leq \mu_{i} f_{i, z}, i=1 \ldots M,
$$

where $\mu_{i}>0$ is the friction coefficient of the contact point $\mathbf{p}_{i}$. The friction cones $K_{1}, \ldots, K_{M} \subseteq \mathbb{R}^{3}$ can be defined as

$$
K_{i}=\left\{\mathbf{x} \in \mathbb{R}^{3} \mid x_{1}^{2}+x_{2}^{2} \leq\left(\mu_{i} x_{3}\right)^{2}, x_{3} \geq 0\right\}, i=1 \ldots M,
$$

and by following this notation, the friction cone constraints of (7) can be formulated as $\mathbf{f}_{i} \in K_{i}, i=1 \ldots M$.

Kinematic constraints: For the kinematic feasibility simplified constraints on the limb lengths relative to the CoM position in global coordinates, denoted by $\mathbf{c} \in \mathbb{R}^{3}$, are defined by

$$
\underline{\mathbf{L}_{\mathbf{i}}} \leq\left\|\mathbf{c}-\mathbf{p}_{i}\right\|_{2} \leq \overline{\mathbf{L}_{\mathbf{i}}}, i=1, \ldots, M .
$$

We define the leg lengths for $\mathbf{p}_{l f}, \mathbf{p}_{r f}$ using $\underline{L}_{l f / r f}=0.64$ and $\bar{L}_{l f / r f}=0.8$ for the stair climbing motion.

\section{B. Objective function}

Before giving the complete formulation of the optimalcontrol problem, we first define the cost terms used for the trajectory optimization over a given time interval. The first term $\ell_{0}$ keeps the CoM close the support foot contacts,

$$
\ell_{0}=\left\|f_{l f}\right\|_{2}^{2} \cdot\left\|c^{x, y}-p_{l f}^{x, y}\right\|_{2}^{2}+\left\|f_{r f}\right\|_{2}^{2} \cdot\left\|c^{x, y}-p_{r f}^{x, y}\right\|_{2}^{2} .
$$

The second term $\ell_{1}$ uses the complementarity (6) to track a reference height depending on the current foot contact height,

$$
\ell_{1}=\left\|\left(\mathbf{f}_{l f, z}+\mathbf{f}_{r f, z}\right)\left(\mathbf{c}_{z}-\mathbf{c}_{z}^{r e f}\right)-\mathbf{f}_{l f, z} \mathbf{p}_{l f, z}-\mathbf{f}_{r f, z} \mathbf{p}_{r f, z}\right\|_{2}^{2} .
$$

The four next terms $\ell_{2}, \ell_{3}, \ell_{4}$ are used to penalize a swaying motion of $\mathbf{c}$ in $z$ direction and stabilize the rotational DoFs.

$$
\ell_{2}=\left\|\dot{\mathbf{c}}_{z}\right\|_{2}^{2}, \ell_{3}=\left\|\omega_{x}\right\|_{2}^{2}, \ell_{4}=\left\|\omega_{y}\right\|_{2}^{2}, \ell_{5}=\left\|\omega_{z}\right\|_{2}^{2} .
$$

The last terms $\ell_{6}$ act as regularization terms,

$$
\ell_{6}=\|\ddot{\mathbf{c}}\|_{2}^{2}+\|\dot{\omega}\|_{2}^{2} .
$$

\section{Optimal control formulation}

We formulate an optimal-control problem to search for the best $\mathrm{CoM}$ trajectory respecting the dynamics (5) and subject to the constraints from Section II-A in terms of a combination of different optimization criteria from Section II-B.

Formulation: The variables of interest are the states and the controls over the time horizon. The state is composed of the CoM position and velocity and angular velocity of the robot: $\mathbf{x}:=(\mathbf{c}, \dot{\mathbf{c}}, \theta, \omega)$. The control is composed of the contact forces for the active contact at time $t$ and the internal angular momentum variation: $\mathbf{u}:=\left(\mathbf{f}_{1}, \ldots, \mathbf{f}_{M}, \sigma\right)$.

The OCP minimizes an objective function of Lagrange type on a finite time horizon $t \in[0, T]$ given by

$$
\begin{array}{rlrl}
\min _{x(\cdot), u(\cdot)} & \int_{0}^{T} l(\mathbf{x}(t), \mathbf{u}(t)) d t & \\
\text { s.t. } & \dot{\mathbf{x}}(t) & =g(\mathbf{x}(t), \mathbf{u}(t)), \quad t \in[0, T], \\
& \mathbf{x}(0)=\mathbf{x}_{0}, \\
& 0 \leq h(\mathbf{x}(t), \mathbf{u}(t)), \quad t \in[0, T] .
\end{array}
$$

where $l(\mathbf{x}, \mathbf{u})=\sum_{j} w_{j} l_{j}(\mathbf{x}, \mathbf{u})$ is the running cost, with a positive weight $w_{j} \in \mathbb{R}$ from Table I adjusting the relative importance and scaling of each term $\ell_{j} ; g: \mathbb{R}^{n_{x}} \times \mathbb{R}^{n_{u}} \rightarrow \mathbb{R}^{n_{x}}$ is representing the dynamics of the system defined in (5); $\mathbf{x}_{0}$ is the initial (measured) state of the system; and $h(\mathbf{x}, \mathbf{u})$ : $\mathbb{R}^{n_{x}} \times \mathbb{R}^{n_{u}} \rightarrow \mathbb{R}^{n_{c}}$ are the mixed state-control path constraints defined by concatenating the friction cone (7), the kinematic constraints (8) as well as the complementarity constraints (6), where the latter is defined via the contact sequence and are not explicitly treated in the OCP. Note that $\dot{\mathbf{p}}_{i}$ are not part of the decision variables of the OCP.

Discretization: Following a direct approach to optimal control, the control $u(\cdot)$ is discretized on a time grid $0=t_{0}<$ $t_{1}<\ldots<t_{K}=T$ by means of base functions parametrized by parameters $\alpha$. We use a piecewise linear discretization which yields smoother CoM trajectories, i.e. for $k=0, \ldots, K-1$ :

$$
\left.u(t)\right|_{\left[\tau_{k}, \tau_{k+1}\right]}:=\left(\alpha_{k, 1}\left(\tau_{k+1}-t\right)+\alpha_{k, 2}\left(t-\tau_{k}\right)\right) /\left(\tau_{k+1}-\tau_{k}\right)
$$

Multiple-shooting resolution: Applying the direct multiple shooting approach for optimal control, we further parametrize the state trajectory $\mathbf{x}(\cdot)$ by solving initial value problems for the differential equation (ODE) (9b) separately on the same grid used for the control discretization. The model dynamics (9b) are adaptively discretized by making use of state-of-the-art ODE solvers. Continuity of the trajectory in the solution of the OCP is enforced by constraints.

From this discretization, a large but structured nonlinear programming problem is obtained that can be solved efficiently with a tailored structure-exploiting sequential quadratic programming (SQP) method [13]. First- and second-order derivatives, required by the SQP method to solve the discretized OCP, involve the computation of sensitivities of the ODE solution according to the principle of internal numerical differentiation (see [14] for details).

\section{Motion Generation}

The computation of the trajectories, depicted in Fig. 2 and needed for the realization of the motion, is a four step 


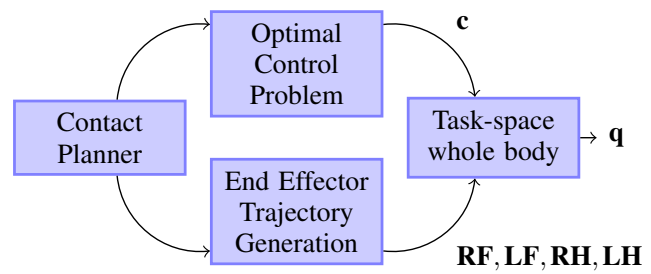

Fig. 2. Overview of the joint trajectories (q) generation process, with $\mathbf{c}$ the CoM trajectory, and RF, LF, RH, LH the end-effector trajectories.

process. (i) A set of contact points has to be found. (ii) The trajectories for the center of mass is computed by the $O C P$. (iii) The full trajectory of the end effectors are computed using B-splines. (iv) The generalized inverse kinematics to compute the actuated joint trajectories of the robot.

In the final process a set of joint trajectories $(\mathbf{q})$ is provided for the position-controlled humanoid robot HRP-2. Note that these trajectories are dynamically consistent and that the contacts are realized according to the predefined schedule of the OCP formulation. The change of angular momentum $\sigma$ was of small effect for the stair climbing motion and was neglected. In cases where $\sigma$ effects the motion, it can be rejected by methods of resolved momentum control using the free limbs during the execution of the motion.

\section{A. Definition of the contact sequence}

For each contact the time interval and the contact state, active or not, is defined. We also specify which body is in contact, the position of the contact, the friction coefficient, and the contact normal vector. The contact sequence is a collection of such contact specifications. Such a contact sequence is typically realized through a contact planner [2], [15]. Here, the contact sequence is predefined, but an extension to additionally optimizing the position of the contacts on a given a set of planar contact surfaces is possible.

\section{B. End-effector trajectories}

In this paper, the hand, the feet and the CoM trajectories are represented using B-splines. In the frame of the Koroibot project, human hand and foot trajectories are studied using inverse optimal control. In addition, meaningful optimization criteria minimized by the under-actuated part of the human dynamics are investigated that will be included in the cost function of problem (9a). At this stage of the project, this is still on-going work and the B-spline parameters are found using heuristics. Although not in the scope of this paper other approaches are possible.

\section{Whole-body generation}

The final whole-body motion is generated by applying the stack-of-tasks (SoT) scheme implementing a generalized inverse kinematics (GIK) as shown in [16]. Given the $\mathrm{CoM}$, the root orientation and end-effector trajectories the SoT framework computes the complete trajectory of all the degrees of freedom of the system. This is done by specifying tasks for the SoT that are defined as a simple PD controller

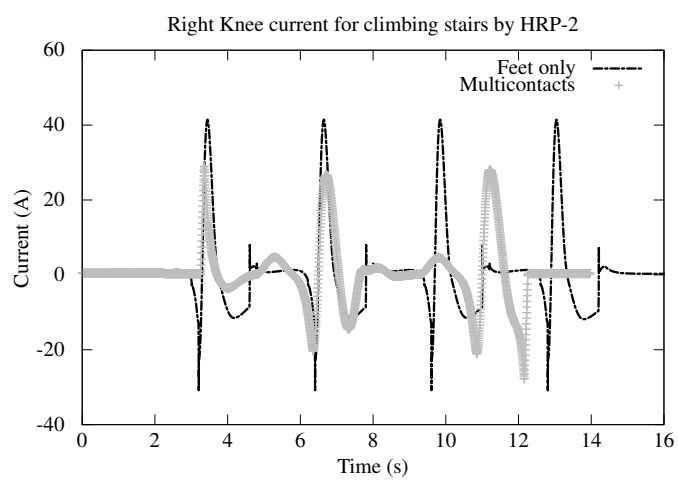

Fig. 6. Current consumption comparison between 2-contacts locomotion and 3-contacts locomotion for climbing a $15 \mathrm{~cm}$ staircase.

tracking the corresponding reference trajectory. The tasks are the following: a task tracking the CoM trajectory along the $x, y, z$ axes $\left(T_{C o m}\right)$, a task for each end-effector position and orientation specification $\left(T_{R H, L H, R F, L F}\right)$, a task to control the orientation of the waist $\left(T_{W}\right)$, and a task regulating the posture of the robot around a nominal posture $\left(T_{q}\right)$. The hierarchy of the tasks defined as the lexicographic order $T_{C o m} \prec T_{R H, L H, R F, L F} \prec T_{W} \prec T_{q}$. The dynamical consistency of the solution with respect to the robot model and the contact forces is implicitly given by the properties of the CoM trajectory computed by the OCP.

The corresponding contact forces and joint torques are then reconstructed with a dynamic simulator. For each time step, the contact forces are computed as the minimal forces corresponding to the joint trajectory $\mathbf{q}, \dot{\mathbf{q}}, \ddot{\mathbf{q}}$ and respecting the contact model by

$$
\min _{\mathbf{f}_{1} \ldots \mathbf{f}_{M}}\left\|R N E A(\mathbf{q}, \dot{\mathbf{q}}, \ddot{\mathbf{q}})-\sum_{i} \mathbf{J}_{i}^{T} \mathbf{f}_{i}\right\|^{2}
$$

such that $\mathbf{f}_{i} \in K_{i}, i=1 . . M$, where $R N E A$ stands for the Recursive Newton Euler Algorithm [17]. The motion can be checked to be dynamically consistent if the residual is null for all time instants of the movement.

\section{RESULTS AND SIMULATION}

\section{Experimental setup}

We have considered the experimental setup depicted in Fig. 1 as proof-of-concept. The goal is to make the humanoid robot HRP-2 climbing a stair case with a hand rail as additional support. The height, the depth, and the width of the stairs are respectively $15 \mathrm{~cm}, 30 \mathrm{~cm}$, and $1 \mathrm{~m}$. The hand rail is a cylinder with a diameter of $3 \mathrm{~cm}$. In this case the handrail is needed to avoid excessive power consumption in the leg motors. Starting and ending in a half-sitting position of the robot, the motion is divided into 3 phases that are executed twice: (i) the right hand establishes contact with the handrail, (ii) the right foot is set on top of the stair in front, (iii) the left foot is lifted and placed next to the right one on the stair. The robot is is in double support phase during the movement of an end-effector and triple support phase during the CoM transition phase. See Fig. 3 for snapshots of the real humanoid robot HRP-2 performing the motion. 


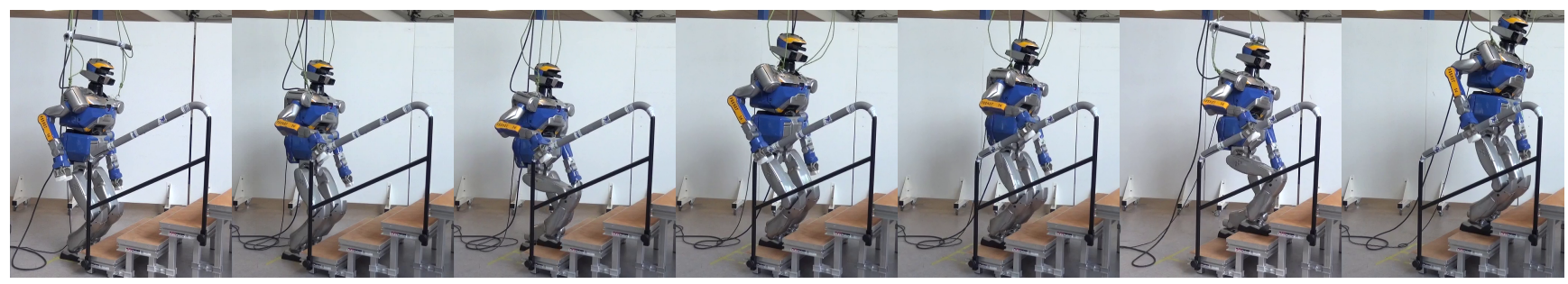

Fig. 3. Set of contact stances realized by the humanoid robot HRP-2 using the method proposed in this paper. The experiment has been realized 5 times in a row without any failure. The companion video shows the realization of the experiment.
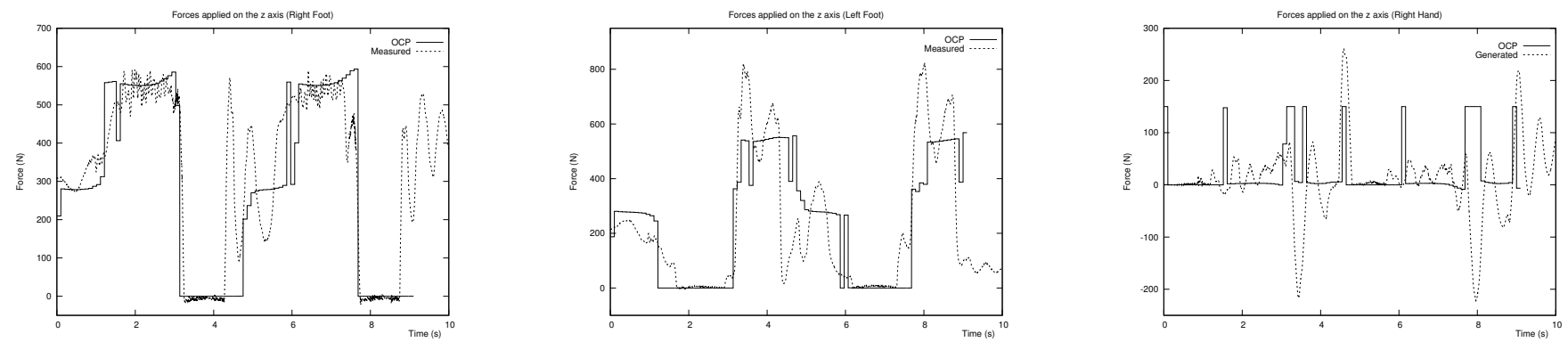

Fig. 4. Measured forces on the HRP-2 humanoid robot during the motion depicted in Fig. 3 compared with the OCP solution.
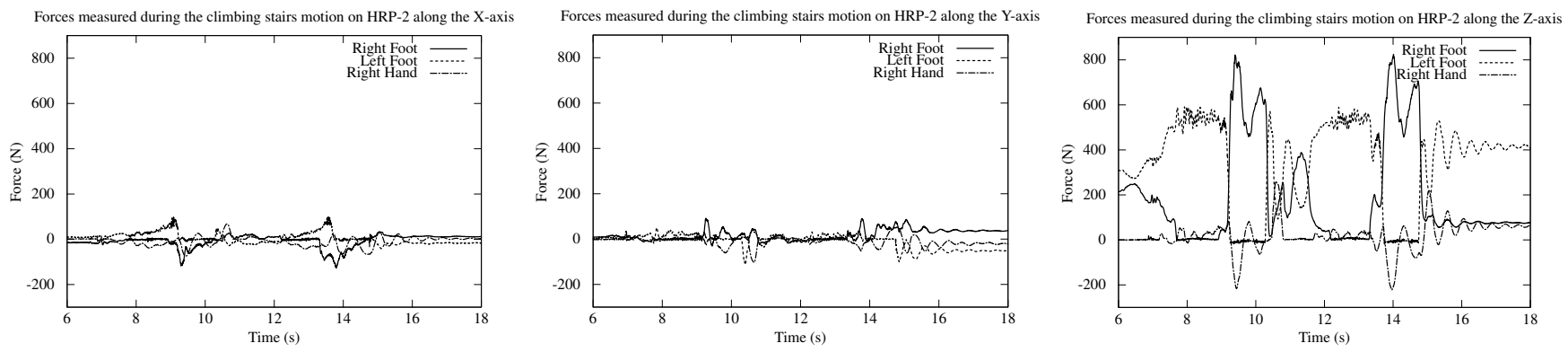

Fig. 5. Measured forces on the HRP-2 humanoid robot during the motion depicted in Fig. 3.

The robot moves an end-effector in $1.4 s$ and moves its center of mass position in $0.1 s$ during a transition phase. The timing of the phase durations is crucial for the robot because they implicitly define the velocity of each limb. From experience in ground-level walking, the period of single support and double support are usually around $0.7 \mathrm{~s}$ and $0.1 s$. However, in this example the robot has to go through a larger distance at each phase than for groundlevel walking. Keeping the same schedule as in ground-level walking makes the robot reaching its actuators limits in speed and current more likely.

The approach presented in this paper has been developed to prove the concept of using a template model for a multicontact controller. In the control chain, only the OCP part is not yet run in real time. In fact the computation time for the motion in the video attachment is $\sim 30$ minutes. The large computational foot print is due to (i) calculating the motion all-at-once on the whole preview-window of $18.4 \mathrm{~s}$, (ii) an over parametrization of the problem (3003 DoFs), (iii) not exploiting the intrinsic sparsity resulting from the template model. Future work will include a tailored implementation of the algorithm considering these bottlenecks and allow a real-time execution on the robot. Despite this, the inverse dynamics run in $1 \mathrm{~ms}$ on the HRP-2 CPU (Intel(R) Core2(TM) Duo E7500, frequency $2.8 \mathrm{GHz}, 1$ core, $3 \mathrm{Mb}$ of cache) under Ubuntu 10.04 LTS.

\section{Forces during contact transition}

Fig. 5 shows the forces measured during one experiment. It appears that almost all the forces are acting along the $z$ axis. The right hand has an important role as it can realize forces up to $200 \mathrm{~N}$ and more remarkable also exerts negative forces at $9 s$ and $14 s$, i.e., the robot also pulls itself utilizing the grip on the handrail. Almost no torque is applied at the level of the feet, the propulsion of the robot is more visible in the tangential forces along the $x$ - and $y$-axis .

Lets now focus on the $z$-axis. At the begin of the motion the robot is stable on its feet and no other contact with its environment is established (phase not shown in the graph). At $6 s$ the hand comes into contact with the handrail causing perturbations in the feet force distribution. The next transition 
appears just before $8 s$. The robot shifts its CoM to the left foot and puts the right foot on the first stair. Then the robot has three contacts with its environment and starts to utilize the hand contact. It pushes with the right leg and pulls with the hand to climb the stair. This particular motion excites the flexibility located under the ankle of HRP-2. Between $10 \mathrm{~s}$ and $12 s$ of the flexibility perturbs the system but the forces on the hand compensate for it. The hand contact stabilizes the robot to be able to move the hand towards the next grasping position at $12 s$, where the robot is back to a stable state again. During the the hand movement, the robot's CoM is effected by the flexibility exertion but not enough to fall down due the stabilizing influence of the grasping contact before and after the double support phase. The motion is repeated once. The only difference is that the hand does not release contact with the handrail at the end of the motion. This helps the robot to stabilize and go back to an equilibrium state.

\section{Current consumption}

A severe limitation in climbing stairs with foot contacts only for human-sized humanoid robot is the current consumption. After performing a large number of experiments on a $15 \mathrm{~cm}$ staircase using a different algorithm [18], it appear that the rate of success was highly dependent on the battery charge level. Based on this observation, and using a model of the robot actuator, the maximum amplitude of the current was detected to be $40 \mathrm{~A}$ on the right knee as depicted in Fig. 6. It is mostly due to the fact that the weight shifting is performed by one support leg. Using several contact points during weight shifting allows to distribute the load across several actuators. Therefore, the current asked for the right knee for the same motion using multiple contacts does not exceed $30 \mathrm{~A}$. This allows performing the motion depicted in Fig. 35 times in a row even with low battery charge level.

\section{CONCLUSION}

In this paper we presented a method to generate a wholebody motion utilizing multiple contact for a humanoid robot. It is based on an optimal control problem formulation focused on the under-actuated part of the robot's dynamics. Combined with state-of-the-art motion-generation algorithms the approach generates feasible trajectories that enable a humanoid robot HRP-2 climbing stairs. The generated motion was performed 5 times in a row on the robot. The current method was not optimized for speed and the next step is to have a real-time feasible implementation on the robot. The approach can also be applied to other kind of motions due to the general formulation, which will be investigated in future work.

\section{ACKNOWLEDGMENTS}

Financial support for the Koroibot project by the European Union within the $7^{\text {th }}$ Framework Programme under Grant Agreement no 611909 is gratefully acknowledged. C. Kirches and M. Kudruss were supported by DFG Graduate School 220 (Heidelberg Graduate School of Mathematical and Computational Methods for the Sciences) funded by the German Excellence Initiative. C. Kirches was supported by the German Federal Ministry of Education and Research program "Mathematics for Innovations in Industry and Service 2013-2016", grant n 05M2013-GOSSIP. O. Stasse was supported by Airbus Future of the Aircraft Factory. We acknowledge all the researchers and trainees who helped during the experiment on HRP-2.

\section{REFERENCES}

[1] H. Dai, A. Valenzuela, and R. Tedrake, "Whole-body motion planning with simple dynamics and full kinematics," in IEEE/RAS Int. Conf. on Humanoid Robotics (ICHR), 2014.

[2] H. Audren, J. Vaillant, A. Kheddar, A. Escande, K. Kaneko, and E. Yoshida, "Model preview control in multi-contact motionapplication to a humanoid robot," in IEEE/RSJ Int. Conf. on Intelligent Robots and Systems (IROS). IEEE, 2014, pp. 4030-4035.

[3] K. H. Koch, K. Mombaur, and P. Soueres, "Optimization-based walking generation for humanoid robot," in SYROCO, 2012.

[4] E. Todorov, "Analytically-invertible dynamics with contacts and constraints: Theory and implementation in MuJoCo," in IEEE/RAS Int. Conf. on Robotics and Automation (ICRA), 2014.

[5] J. Koenemann, A. Del Prete, Y. Tassa, E. Todorov, O. Stasse, M. Bennewitz, and N. Mansard, "Whole-body Model-Predictive Control applied to the HRP-2 Humanoid," in IEEE/RSJ Int. Conf. on Intelligent Robots and Systems (IROS), 2015.

[6] H. Hirukawa, S. Hattori, S. Kajita, K. Harada, K. Kaneko, F. Kanehiro, M. Morisawa, and S. Nakaoka, "A pattern generator of humanoid robots walking on a rough terrain," in IEEE/RAS Int. Conf. on Robotics and Automation (ICRA), 2007, pp. 2181-2187.

[7] C. Ott, M. A. Roa, and G. Herzinger, "Posture and balance control for biped robots based on contact force optimization," in IEEE/RAS Int. Conf. on Humanoid Robotics (ICHR), 2011, pp. 26-33.

[8] S. Hyon, J. G. Hale, and G. Cheng, "Full-body compliant humanhumanoid interaction: Balancing in the presence of unknown external forces," IEEE Transactions on Robotics, vol. 23, no. 5, pp. 884-898, 2007.

[9] K. Nishiwaki, J. E. Chestnutt, and S. Kagami, "Autonomous navigation of a humanoid robot over unknown rough terrain using a laser range sensor," The International Journal of Robotics Research, vol. 31, no. 11, pp. 1251-1262, 2012.

[10] K. Kaneko, F. Kanehiro, S. Kajita, H. Hirukawa, T. Kawasaki, M. Hirata, K. Akachi, and T. Isozumi, "Humanoid robot hrp-2," in IEEE/RAS Int. Conf. on Robotics and Automation (ICRA), vol. 2, 2004, pp. 10831090.

[11] D. E. Orin, A. Goswami, and S.-H. Lee, "Centroidal dynamics of a humanoid robot," Autonomous Robots, vol. 35, no. 2-3, pp. 161-176, 2013.

[12] S.-P. Boyd and B. Wegbreit, "Fast computation of optimal contact forces," IEEE Transactions on Robotics, vol. 23, no. 6, pp. 11171132, 2007

[13] D. B. Leineweber, I. Bauer, H. G. Bock, and J. P. Schlöder, "An efficient multiple shooting based reduced SQP strategy for large-scale dynamic process optimization. Part 1: Theoretical aspects," Computers \& Chemical Engineering, vol. 27, no. 2, pp. 157-166, 2003.

[14] H. G. Bock, Numerical treatment of inverse problems in chemical reaction kinetics. Springer, 1981.

[15] S. Tonneau, N. Mansard, C. Park, D. Manocha, F. Multon, and J. Pettré, "A reachability-based planner for sequences of acyclic contacts in cluttered environments," in International Symposium of Robotics Research (ISRR), Genova, Italy, October 2015.

[16] N. Mansard, O. Stasse, P. Evrard, and A. Kheddar, "A versatile generalized inverted kinematics implementation for collaborative working humanoid robots: The stack of tasks," in International Conference on Advanced Robotics (ICAR), June 2009, p. 119.

[17] J. Y. Luh, M. W. Walker, and R. P. Paul, "On-line computational scheme for mechanical manipulators," Journal of Dynamic Systems, Measurement, and Control, vol. 102, no. 2, pp. 69-76, 1980.

[18] M. Morisawa, K. Harada, S. Kajita, S. Nakaoka, K. Fujiwara, F. Kanehiro, K. Kaneko, and H. Hirukawa, "Experimentation of humanoid walking allowing immediate modification of foot place based on analytical solution," in IEEE/RAS Int. Conf. on Robotics and Automation (ICRA), 2007, pp. 3989-3994. 